\title{
Borromean Supercounterfluidity
}

\author{
Emil Blomquist@, Andrzej Syrwid@, and Egor Babaev \\ Department of Physics, KTH Royal Institute of Technology, Stockholm SE-10691, Sweden
}

(Received 6 September 2021; accepted 12 November 2021; published 15 December 2021)

\begin{abstract}
We demonstrate microscopically the existence of a new superfluid state of matter in a three-component Bose mixture trapped in an optical lattice. The superfluid transport involving coflow of all three components is arrested in that state, while counterflows between any pair of components are dissipationless. The presence of three components allows for three different types of counterflows with only two independent superfluid degrees of freedom.
\end{abstract}

DOI: 10.1103/PhysRevLett.127.255303

The advent of optical lattices [1-6] allowed for highly controllable access to strongly correlated quantum manybody systems and opened up a way to realize various phases of matter. One of the theoretical predictions was that bosons in optical lattices could host a new type of transport phenomenon called supercounterfluidity [7-16]. That is, having two bosonic fields $\psi_{1,2}$, in an ordinary case, one finds a superfluid mixture when $\left\langle\psi_{1,2}\right\rangle$ reveals a (quasi) long-range order. This state is predicted to appear [7-16] when the averages of individual fields vanish, $\left\langle\psi_{1,2}\right\rangle=0$, but there is a (quasi) long-range order in the composite field $\left\langle\psi_{1} \psi_{2}^{*}\right\rangle \neq 0$. Therefore, in a supercounterfluid phase, individual bosonic species do not exhibit superfluidity, but the transport of particle-hole composites is dissipationless. A similar type of order was predicted in superconducting systems arising from a different microscopic origin [17-24]. For a general discussion, see [25]. Recently, an experimental observation of a discrete-symmetry counterpart of this type of order was reported in a threecomponent superconductor [26]. In the two-component case, the order parameter for the supercounterfluid is partially similar to a condensate of bound particle-hole pairs between two different components. However, the three-component situation is more subtle. In this Letter, we demonstrate microscopically the existence of a new "super" state in a three-component Bose mixture.

At the superfluid hydrodynamic level, $N$-component isotropic superfluid systems can in general be described by the free-energy density $f=\frac{1}{2} \sum_{\alpha, \beta} \rho_{\alpha \beta} \mathbf{v}_{\alpha} \cdot \mathbf{v}_{\beta}$. Here, $\mathbf{v}_{\alpha}=\nabla \theta_{\alpha} / m_{\alpha}(\hbar=1)$ represents the superfluid velocity of component $\alpha$, where $\theta_{\alpha} \in[0,2 \pi)$ and $m_{\alpha}$ denote superfluid phase and particle mass of the $\alpha$ th component,

Published by the American Physical Society under the terms of the Creative Commons Attribution 4.0 International license. Further distribution of this work must maintain attribution to the author(s) and the published article's title, journal citation, and DOI. Funded by Bibsam. respectively. In the presence of intercomponent interactions, the superfluid stiffness tensor $\rho$ will in addition to the diagonal superfluid density elements $\rho_{\alpha \alpha}$ also contain off-diagonal elements $\rho_{\alpha \beta}(\alpha \neq \beta)$, coupling the different components [27]. This in turns has principal consequences for the corresponding superflows $\mathbf{j}_{\alpha}=\partial f / \partial \mathbf{v}_{\alpha}=\rho_{\alpha \alpha} \mathbf{v}_{\alpha}+$ $\sum_{\beta \neq \alpha} \rho_{\alpha \beta} \mathbf{v}_{\beta}$. Namely, the superflow of one component can be induced by the flow of a different component. This fundamental phenomenon is referred to as AndreevBashkin drag [27]. The Andreev-Bashkin effect can be well controlled in optical lattices [7,8,11,16,28-30]. Note that in certain asymmetrical optical lattices there are additional terms responsible for transverse entrainment [31]. However, in this Letter, we will restrict ourselves to square lattices where only the Andreev-Bashkin drag effect is present.

In the simplest case of two components with identical masses, the free-energy density can be cast into the form associated with coflows and counterflows $f=$ $\rho_{2}\left(\nabla \theta_{1}+\nabla \theta_{2}\right)^{2} / 4+\rho_{0}\left(\nabla \theta_{1}-\nabla \theta_{2}\right)^{2} / 4$. Here, $\rho_{\xi}=\rho_{k}+$ $(\xi-1) \rho_{d} \geq 0$ where $\rho_{k}>0$ describe the prefactor of the standard gradient term, and $\rho_{d}$-either positive or negative - denotes the drag strength. When the drag is sufficiently strong and negative, the cheapest topological excitations become cocirculating composite vortices, i.e., vortices where both phases $\theta_{1,2}$ wind by $2 \pi$ around the core [25]. Thermal or quantum fluctuations can then lead to the proliferation of these composite vortices-but not elementary ones-resulting in a phase transition to a supercounterfluid (for a detailed discussion of the principle, see, e.g., Chap. 6 in [25]). The composite vortices do not induce gradients in the phase difference and thus do not disorder the phase difference part of the free energy. The free-energy density of the resulting state therefore only involves the phase stiffness corresponding to the phase difference, i.e., $f=\rho_{0}\left(\nabla \theta_{1}-\nabla \theta_{2}\right)^{2} / 4$. This term can be interpreted as the kinetic free-energy contribution related to the composite particle-hole order parameter $\psi_{1} \psi_{2}^{*}$. 
Consequently, only counterflow dissipationless transport can take place.

In a system with more than two components, states may arise with no direct counterpart among two-component superfluids. Let us therefore consider a two-dimensional $\mathrm{N}$-component symmetric quantum system, i.e., components with identical masses and densities, and equal Andreev-Bashkin drag strength $\rho_{d}$ between each pair. We start with a phase-only approximation assuming identical and homogeneous densities of unit mass particles $\left(m_{\alpha}=1\right)$ in each superfluid component. The corresponding free-energy density reads

$$
\begin{aligned}
f & =\frac{\rho_{k}}{2} \sum_{\alpha}\left(\nabla \theta_{\alpha}\right)^{2}+\frac{\rho_{d}}{2} \sum_{\alpha, \beta \neq \alpha} \nabla \theta_{\alpha} \cdot \nabla \theta_{\beta} \\
& =\frac{\rho_{N}}{2 N}\left(\sum_{\alpha} \nabla \theta_{\alpha}\right)^{2}+\frac{\rho_{0}}{4 N} \sum_{\alpha, \beta}\left(\nabla \theta_{\alpha}-\nabla \theta_{\beta}\right)^{2},
\end{aligned}
$$

where again $\rho_{\xi}=\rho_{k}+(\xi-1) \rho_{d} \geq 0$. Now, when the drag is strong and negative, i.e., when $\rho_{N} \ll \rho_{0}$, the cheapest topological excitations are composite vortices where all $N$ phases wind by $2 \pi$ with the same orientation. Consequently, the proliferation of the three-component topological defects leads to a state where the sum of three phases is disordered. However, these composite vortices are unable to disorder phase differences and the system retains $N-1$ superfluid modes. Equation (1) therefore reduces to $f \propto \sum_{\alpha, \beta}\left(\nabla \theta_{\alpha}-\right.$ $\left.\nabla \theta_{\beta}\right)^{2}$ and the corresponding phase is characterized by zero net superflow, i.e., $\sum_{\alpha} \mathbf{j}_{\alpha} \propto \sum_{\alpha} \partial f / \partial\left(\nabla \theta_{\alpha}\right)=\mathbf{0}$.

Consequently, for $N>2$, the transport properties of the new phase can be understood as a counterflow of two components where the presence of the third symmetric component allows for fluctuations in the type of counterpropagating companions. One would anticipate that this should be reflected in the world lines of the microscopic path-integral formulation. That is, in different regions of the system, one should find different types of particle-hole paired world lines. Moreover, there is no superfluid coflow of bound $N$ particle states, while counterpropagation of any two different components is dissipationless. Specifically, for $N=3$ where $\alpha \in\{r, g, b\}$, there are three types of counterflows for which there are only two independent degrees of freedom. That implies that we can have a superfluid coflow of two components as long as their combined flow is counteracted by the flow of the third component, e.g., $\mathbf{j}_{r}=\mathbf{j}_{g}=\mathbf{j}$ and $\mathbf{j}_{b}=-2 \mathbf{j}$. Here, we can draw a distant analogy to the Borromean rings where three rings are confined while each pair of rings is deconfined, see Fig. 1. Hence, we coin this phenomenon Borromean supercounterfluidity.

Below, we microscopically demonstrate that such a superfluid state exists in a three-component $(N=3)$ Bose-Hubbard model [32].

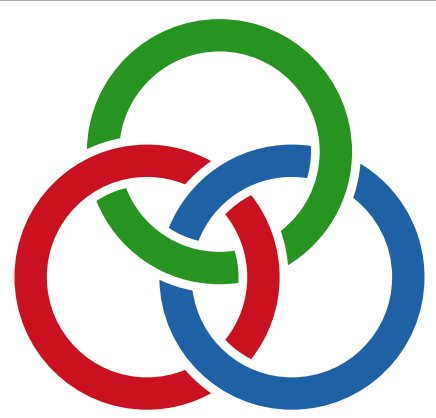

FIG. 1. The Borromean rings. If a single ring is removed, the two remaining ones will become unlinked.

$$
\begin{aligned}
\hat{H}= & -t \sum_{\alpha} \sum_{\langle i j\rangle} \hat{b}_{i \alpha}^{\dagger} \hat{b}_{j \alpha}+\frac{U}{2} \sum_{\alpha} \sum_{i} \hat{n}_{i \alpha}\left(\hat{n}_{i \alpha}-1\right) \\
& +\frac{U^{\prime}}{2} \sum_{\alpha, \beta \neq \alpha} \sum_{i} \hat{n}_{i \alpha} \hat{n}_{i \beta} .
\end{aligned}
$$

Here, $\hat{b}_{i \alpha}\left(\hat{b}_{i \alpha}^{\dagger}\right)$ is the bosonic annihilation (creation) operator of component $\alpha$ at site $i$, and $\hat{n}_{i \alpha}=\hat{b}_{i \alpha}^{\dagger} \hat{b}_{i \alpha}$ is the corresponding particle-number operator. Greek subscripts label the component type, i.e., $r$ (red), $g$ (green), and $b$ (blue). The parameter $t$ represents the hopping amplitude, while $U$ and $U^{\prime}$, respectively, are the intracomponent and intercomponent on-site interaction strengths. We will consider a $L \times L$ square lattice with unit lattice constant and periodic boundary conditions. We further analyze the two separate cases where either the individual particle-number densities are fixed, i.e., $n_{\alpha}:=\left\langle\sum_{i} \hat{n}_{i \alpha}\right\rangle / L^{2}=1 / 3$, or the total particle-number density is conserved, i.e., $\sum_{\alpha} n_{\alpha}=1$, while allowing for fluctuations in $n_{\alpha}$.

We numerically investigate the system by utilizing worm-algorithm Monte Carlo [33-37]-a quantum Monte-Carlo method which samples path-integral configurations of the partition function in real space and imaginary time. To extract the numerical values of $\rho_{k}$ and $\rho_{d}$ appearing in the free-energy density, Eq. (1), we generalize Pollock and Ceperley's formula [16,31,38]: $\rho_{k}=T\left\langle\mathbf{w}_{\alpha}^{2}\right\rangle / 2$, and $\rho_{d}=T\left\langle\mathbf{w}_{\alpha} \cdot \mathbf{w}_{\beta}\right\rangle / 2$ where $T$ is the temperature $\left(k_{B}=1\right)$ and $\alpha \neq \beta$. The winding numbers $\mathbf{w}_{\alpha}$ encode the net number of times, and in which direction, $\alpha$-type particles cross the periodic boundaries. The notation $\langle\cdot\rangle$ refers to the standard statistical Monte Carlo average.

The calculated coefficients $\rho_{k}, \rho_{d}$, and their ratios $\rho_{d} / \rho_{k}$ are presented in Fig. 2 as functions of $t / U$ for the interaction strengths $U=1$ and $U^{\prime}=0.9$. For small $t / U$ we observe $\rho_{k}=\rho_{d}=0$, which indicates a Mott insulating phase. However, at $t / U \gtrsim 0.02$ the system enters the Borromean supercounterfluid phase, where $\rho_{d} / \rho_{k}$ rapidly saturates at the value $-1 / 2$ for which the coefficient $\rho_{N=3}$ in Eq. (1) vanishes. This result demonstrates that the corresponding effective free-energy density is given by gradients of phase differences between all the three components. 


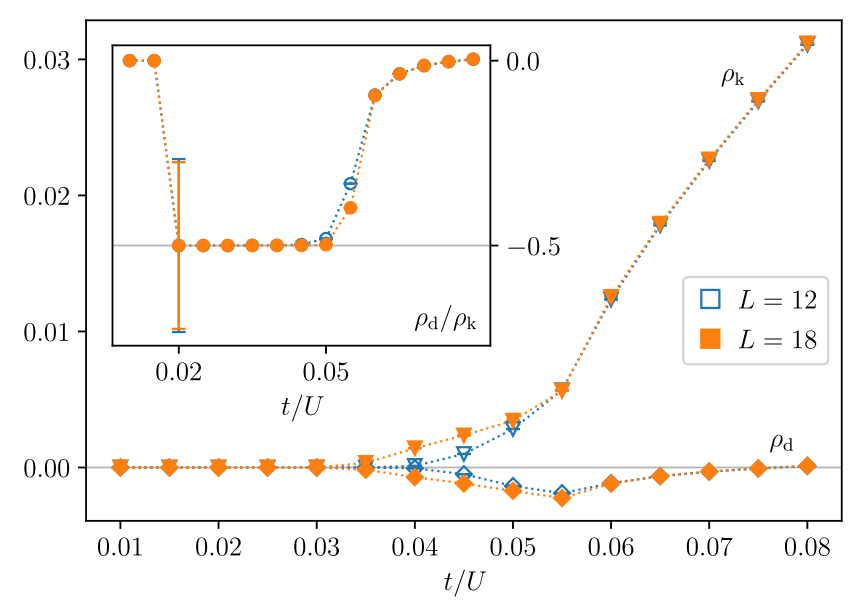

FIG. 2. The superfluid density $\rho_{k}$ (triangles), the AndreevBashkin drag-coefficient $\rho_{d}$ (diamonds), and the ratio $\rho_{d} / \rho_{k}$ (inset) as a function of $t / U$. For $t / U \in[0.02,0.05]$ the ratio $\rho_{d} / \rho_{k}$ saturates to its lower bound, $-1 / 2$, which indicates the presence of the Borromean supercounterfluid phase. The computations were performed using $U=1, U^{\prime}=0.9, T=t / L, L=12,18$, and a fixed particle-number density $n_{\alpha}=1 / 3$.

When further increasing $t / U \gtrsim 0.05$, the system undergoes a second transition to the three-component superfluid phase where the $U(1) \times U(1) \times U(1)$ symmetry is broken. Deep in this regime $\rho_{d} / \rho_{k}$ becomes very small.

Let us now characterize the absence of phase separation in the system. Namely, suppose the system spontaneously forms bound pairs between two components only, and one relaxes the restriction on individually fixed particle numbers $n_{\alpha}$. In that case, a disproportion between the components' particle densities is expected. To that end, we investigate the density imbalance $\Delta n$ defined through

$$
\left\langle\hat{n}_{r} \hat{n}_{g} \hat{n}_{b}\right\rangle=(1 / 3-2 \Delta n)(1 / 3+\Delta n)^{2},
$$

while constraining the total particle-number density to $n_{r}+n_{g}+n_{b}=1$, and leaving $n_{\alpha}$ unrestricted such that only on average $\left\langle\hat{n}_{\alpha}\right\rangle=1 / 3$. Here, $\hat{n}_{\alpha}=\sum_{i} \hat{n}_{i \alpha} / L^{2}$. If the particles of one component are completely exchanged in favor of particles belonging to the other two components, then $\left\langle\hat{n}_{r} \hat{n}_{g} \hat{n}_{b}\right\rangle=0$ leading to $\Delta n=1 / 6$. Similarly, if two components are completely expelled then $\Delta n=-1 / 3$. In contrast, if there is no density imbalance and all components are equally represented one should have $\left\langle\hat{n}_{r} \hat{n}_{g} \hat{n}_{b}\right\rangle=$ $(1 / 3)^{3}$ such that $\Delta n=0$. In Fig. 3(a) we demonstrate a decay of $\Delta n$ with increased system size $L$ and inverse temperature $\beta$ in the regime of the anticipated Borromean supercounterfluid phase. As expected, we find a nonzero $\Delta n$ at finite temperatures due to thermal density fluctuations, however, the magnitude of $\Delta n$ is small in comparison to $1 / 3$. In addition, $\Delta n$ show a clear decay with increased $L$ indicating $\Delta n \rightarrow 0$ for $L \rightarrow \infty$. This is further corroborated in Figs. 3(b) and 3(c) by real-space particle-number

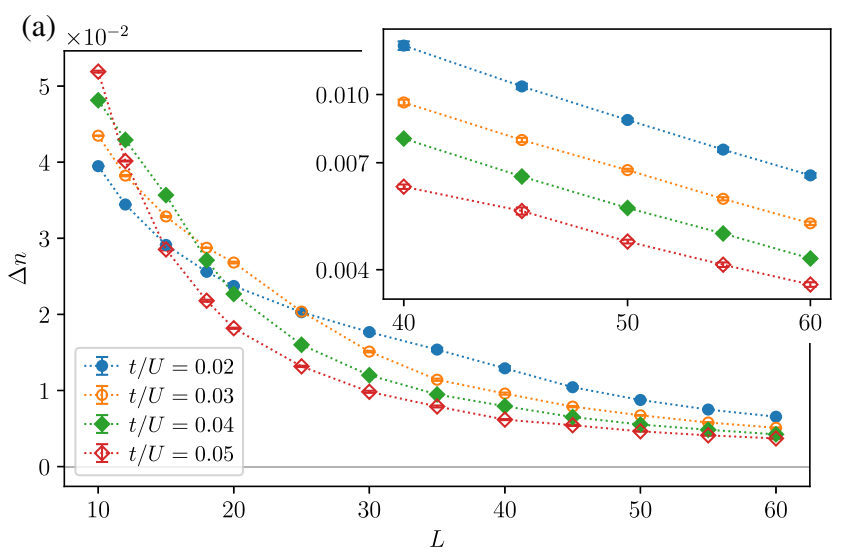

(b)

(c)
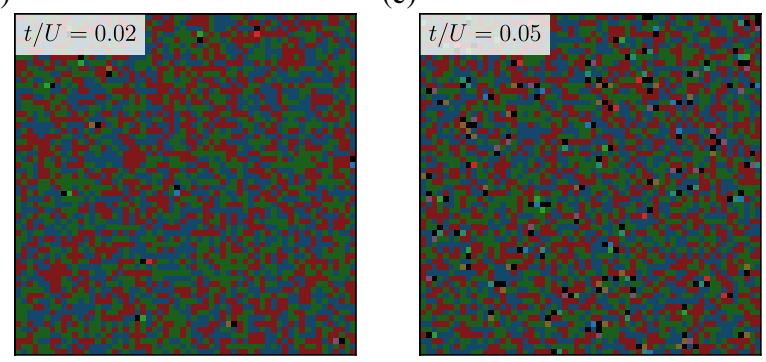

FIG. 3. In panel (a) the particle-number-density imbalance $\Delta n$, Eq. (3), is plotted versus the system size $L$ for various parameter values $t / U$ with the inverse temperature given by $\beta=L / t$. The total particle-number-density $n_{r}+n_{g}+n_{b}=1$ is fixed while the particle number of each of the three symmetric components is allowed to fluctuate. The plot reveals a decay of $\Delta n$ with increasing $L$. The latter is more apparent in the inset which presents $\Delta n$ for the largest $L$ 's on logarithmic scales. This shows that $\Delta n \rightarrow 0$ as $L \rightarrow \infty$. The bottom panels present two representative real-space particle-number distributions, i.e., imaginarytime slices of the world-line configuration, with $L=60$ for $t / U=0.02$ (b) and $t / U=0.05$ (c). The three different components are represented by the colors red, green, and blue, respectively. The darker the color, the fewer the particles occupying the corresponding site such that an empty site becomes black. These particle-number distributions clearly display a miscible phase which is corroborated by $\Delta n$ of panel (a). In all cases the values $U=1$ and $U^{\prime}=0.9$ were used.

distributions obtained from two representative imaginarytime slices of the world-line configurations. This demonstrates that there is no density imbalance nor phase separation in the ground state.

The Borromean supercounterfluid phase can be further studied by inspecting typical partition function world-line configurations sampled with the help of the wormalgorithm Monte Carlo method. A representative configuration of the Borromean superfluid phase is illustrated in Fig. 4, which indeed reveals three mixed components. The net flow of particles in imaginary time further exhibits the counterflowlike behavior, resulting in the winding numbers $\left\langle\mathbf{w}_{\alpha}^{2}\right\rangle \neq 0$ and $\left\langle\left(\sum_{\alpha} \mathbf{w}_{\alpha}\right)^{2}\right\rangle=0$, or alternatively $\rho_{d} / \rho_{k}=-1 / 2$. 


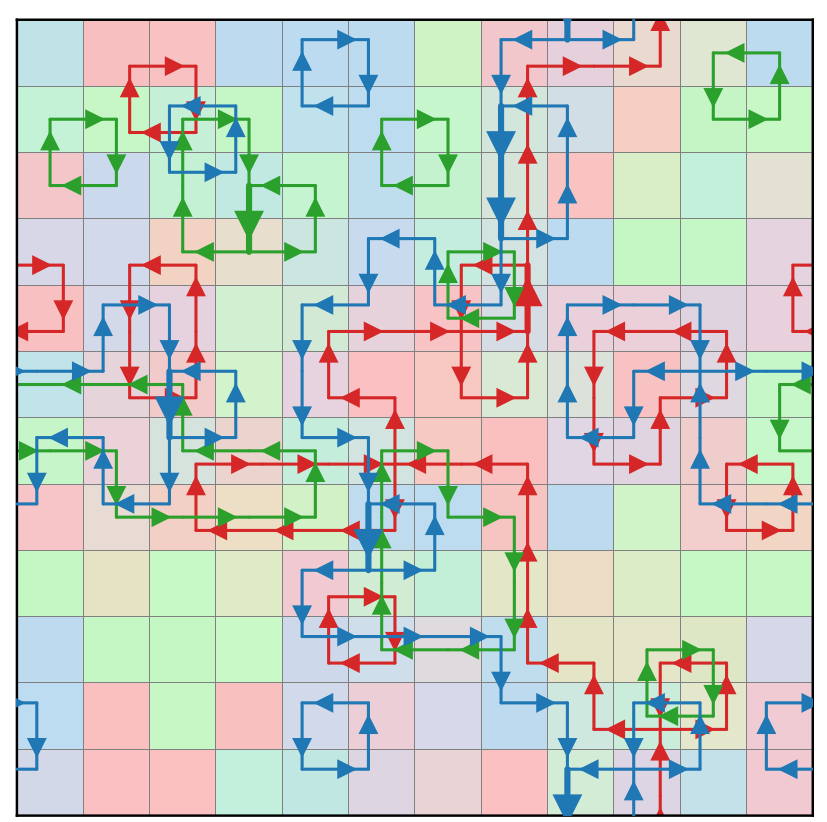

FIG. 4. Spatial projection of a typical partition function worldline configuration of the Borromean supercounterfluid phase. Here, the three different components are represented by red, green, and blue, respectively. The directional loops indicate the net particle propagation in imaginary time between adjacent sites, and the site's color indicates its average particle-number population. The sites reveal three miscible components, and the loops display on average counterpropagating world-line trajectories. These properties are indicative of the Borromean supercounterfluid phase. By counting the flux of loops across the boundaries we find the winding numbers $\mathbf{w}_{r}=\mathbf{e}_{y}, \mathbf{w}_{g}=\mathbf{0}, \mathbf{w}_{b}=-\mathbf{e}_{y}$ which satisfy $\mathbf{w}_{r}+\mathbf{w}_{g}+\mathbf{w}_{b}=\mathbf{0}$. The results were obtained using $t=0.04, \quad U=1, \quad U^{\prime}=0.9, L=12, T=t / L$, and a fixed particle-number density $n_{\alpha}=1 / 3$.

In conclusion, we have demonstrated microscopically that a strongly correlated three-component bosonic mixture - realizable in optical lattice setups - has a phase with "super" transport phenomenon different from conventional superfluidity. In this phase, the simultaneous coflow of all three bosonic components is arrested, while the system retains dissipationless counterflows between any pair of components. These three counterflows are not independent but rather described by two superfluid degrees of freedom. At the microscopic level, the types of counterpropagating partners can vary.

Possible realization of these states in optical lattices could be obtained by trapping mixtures of bosonic isotopes of $\mathrm{Na}$ and $\mathrm{K}$. Possible ways to detect the Borromean supercounterfluid state experimentally is through tilting the lattice and detecting the ratio between transport of different individual components. However, the more striking signature can be obtained by observing a dramatic change in the system's rotational response. Namely, a rotating conventional superfluid can be described by introducing a fictitious vector potential $\boldsymbol{\Theta}$, leading to a vortex lattice formation (see, e.g., Chap. 1 in [25]). In the Borromean supercounterfluid state the vector potential $\boldsymbol{\Theta}$ couples to the phase gradients through mass differences according to $f \approx \sum_{\alpha, \beta \neq \alpha}\left[\nabla \theta_{\alpha}-\nabla \theta_{\beta}-\left(m_{\alpha}-m_{\beta}\right) \boldsymbol{\Theta}\right]^{2}$. If all the components have equal particle masses, i.e., $m_{\alpha}=m_{\beta}$, the system is unaffected by rotation. Indeed, in that case, the counterflow involves no mass transfer, and vortices carry no angular momentum. On the other hand, if $m_{\alpha} \neq m_{\beta}$ the system forms a vortex lattice to compensate for the superextensive increase of the free energy due to the rotation. In the latter case, the critical velocities and number of rotation-induced vortices will be proportional to the mass difference $m_{\alpha}-m_{\beta}$ rather than to masses of individual components.

We would like to thank Martin Zwierlein and Immanuel Bloch for discussions on experimental realizations of bosonic mixtures. E. Bl. and E. Ba. were supported by the Swedish Research Council Grants No. 2016-06122, No. 2018-03659, and Göran Gustafsson Foundation for Research in Natural Sciences. A. S. and E. Ba. acknowledge the support from Olle Engkvist Foundation. The computations were enabled by resources provided by the Swedish National Infrastructure for Computing (SNIC) at the National Supercomputer Centre (NSC) partially funded by the Swedish Research Council through Grant No. 2018-05973.

[1] D. Jaksch, C. Bruder, J. I. Cirac, C. W. Gardiner, and P. Zoller, Phys. Rev. Lett. 81, 3108 (1998).

[2] D. Jaksch and P. Zoller, Ann. Phys. (Amsterdam) 315, 52 (2005), special issue.

[3] M. Greiner, O. Mandel, T. Esslinger, T. W. Hänsch, and I. Bloch, Nature (London) 415, 39 (2002).

[4] I. Bloch, Nat. Phys. 1, 23 (2005).

[5] I. Bloch, J. Dalibard, and W. Zwerger, Rev. Mod. Phys. 80, 885 (2008).

[6] M. Lewenstein, A. Sanpera, V. Ahufinger, B. Damski, A. Sen, and U. Sen, Adv. Phys. 56, 243 (2007).

[7] A. B. Kuklov and B. V. Svistunov, Phys. Rev. Lett. 90, 100401 (2003).

[8] A. Kuklov, N. Prokof'ev, and B. Svistunov, Phys. Rev. Lett. 92, 050402 (2004).

[9] A. Kuklov, N. Prokof'ev, and B. Svistunov, Phys. Rev. Lett. 92, 030403 (2004).

[10] A. Kuklov, N. Prokof'ev, B. Svistunov, and M. Troyer, Ann. Phys. (Amsterdam) 321, 1602 (2006).

[11] B. Capogrosso-Sansone, Ş. G. Söyler, N. Prokof'ev, and B. Svistunov, Phys. Rev. A 77, 015602 (2008).

[12] E. K. Dahl, E. Babaev, S. Kragset, and A. Sudbø, Phys. Rev. B 77, 144519 (2008).

[13] E. K. Dahl, E. Babaev, and A. Sudbø, Phys. Rev. Lett. 101, 255301 (2008).

[14] E. K. Dahl, E. Babaev, and A. Sudbø, Phys. Rev. B 78, 144510 (2008). 
[15] B. Capogrosso-Sansone and A. Kuklov, J. Low Temp. Phys. 165, 213 (2011).

[16] K. Sellin and E. Babaev, Phys. Rev. B 97, 094517 (2018).

[17] E. Babaev, arXiv:cond-mat/0201547.

[18] E. Babaev, A. Sudbø, and N. Ashcroft, Nature (London) 431, 666 (2004).

[19] J. Smiseth, E. Smørgrav, E. Babaev, and A. Sudbø, Phys. Rev. B 71, 214509 (2005).

[20] E. V. Herland, E. Babaev, and A. Sudbø, Phys. Rev. B 82, 134511 (2010).

[21] A. B. Kuklov, M. Matsumoto, N. V. Prokof'ev, B. V. Svistunov, and M. Troyer, Phys. Rev. Lett. 101, 050405 (2008).

[22] D. Agterberg and H. Tsunetsugu, Nat. Phys. 4, 639 (2008).

[23] E. Berg, E. Fradkin, and S. A. Kivelson, Nat. Phys. 5, 830 (2009).

[24] D. Weston and E. Babaev, Phys. Rev. B 104, 075116 (2021).

[25] B. V. Svistunov, E. S. Babaev, and N. V. Prokof'ev, Superfluid States of Matter (CRC Press, Boca Raton, 2015).

[26] V. Grinenko, D. Weston, F. Caglieris et al., Nat. Phys. 17, 1254 (2021).

[27] A. F. Andreev and E. P. Bashkin, Zh. Eksp. Teor. Fiz. 69, 319 (1975) [Sov. Phys. JETP 42, 164 (1975)], http://jetp.ras .ru/cgi-bin/e/index/e/42/1/p164?a=list.
[28] J. Linder and A. Sudbø, Phys. Rev. A 79, 063610 (2009).

[29] P. P. Hofer, C. Bruder, and V. M. Stojanović, Phys. Rev. A 86, 033627 (2012).

[30] D. Contessi, D. Romito, M. Rizzi, and A. Recati, Phys. Rev. Research 3, L022017 (2021).

[31] A. Syrwid, E. Blomquist, and E. Babaev, Phys. Rev. Lett. 127, 100403 (2021).

[32] H. A. Gersch and G. C. Knollman, Phys. Rev. 129, 959 (1963).

[33] N. Prokof'ev, B. Svistunov, and I. Tupitsyn, Phys. Lett. A 238, 253 (1998).

[34] B. Capogrosso-Sansone, N. V. Prokof'ev, and B. V. Svistunov, Phys. Rev. B 75, 134302 (2007).

[35] E. Blomquist, Strong correlation effects in bosonic and fermionic systems through an unbiased quantum Monte Carlo approach, Ph.D. thesis, KTH, Condensed Matter Theory, 2021.

[36] K. Sellin, Structure formation, phase transitions and drag interactions in multicomponent superconductors and superfluids, Ph.D. thesis, KTH, Statistical Physics, 2018.

[37] F. Lingua, B. Capogrosso-Sansone, A. Safavi-Naini, A. Jahangiri, and V. Penna, Phys. Scr. 93, 105402 (2018).

[38] E. L. Pollock and D. M. Ceperley, Phys. Rev. B 36, 8343 (1987). 